\title{
Impacto da ausência de compatibilização de projetos na execução de uma obra residencial
}

\section{Impact of the absence of compatibility of projects in the execution of residential construction}

Article Info:

Article history: Received 2021-03-29 / Accepted 2021-03-29 / Available online 2021-03-30

doi: 10.18540/jcecvl7iss1pp11922-01-09e

Lorran Nunes Gomes

ORCID: https://orcid.org/0000-0001-7249-1388

Departamento de Engenharia Civil, Núcleo de Tecnologia, Universidade Federal de Rondônia,

Brasil

E-mail: lorranngomes@gmail.com

Diego Henrique de Almeida

ORCID: http://orcid.org/0000-0002-6220-4004

Departamento de Engenharia Civil, Núcleo de Tecnologia, Universidade Federal de Rondônia,

Brasil

E-mail: diegoalmeida@unir.br

\section{Resumo}

Para execução de uma obra residencial é necessário primeiramente a etapa de desenvolvimento de projetos, projetando cada disciplina sequencialmente ou paralelamente. A interação de mais de um profissional na elaboração de um projeto é o caminho certo para uma boa elaboração, entretanto, a centralização de dados permite melhor gestão das informações mitigando problemas de incompatibilidade, que refletem na separação sequencial dos projetos, uma vez que compatibilização fornece uma visão holística do processo. O objetivo deste artigo é analisar erros de execução na construção de uma edificação habitacional em estruturas de concreto armado com lajes pré-moldadas de concreto e vedação em alvenaria, devido à incompatibilidade entre projetos. Para o desenvolvimento da pesquisa foi desenvolvido o acompanhamento das fases de construção por meio de visitas técnicas regulares e registro fotográfico. Sendo uma obra de pequeno porte, os projetos foram realizados de maneira independente e sem o estudo prévio das etapas da construção, o que favoreceu as inconformidades neste estudo de caso. Foram verificados erros de execução causados por incompatibilidade entre projetos arquitetônico, estrutural, elétrico e hidráulico que impactaram negativamente durante a execução do projeto. Desta maneira ressalta-se a importância da compatibilidade entre projetos independente do porte da obra.

Palavras-chave: Compatibilização. Construção Civil. Incompatibilidade. Projeto.

\section{Abstract}

For the execution of a residential project, it is necessary first the project development stage, designing each discipline sequentially or in parallel. The interaction of more than one professional in the elaboration of a project is the right way for a good elaboration, however, the centralization of data allows better management of the information, mitigating incompatibility problems, which reflect in the sequential separation of the projects, since compatibilization provides a holistic view of the process. The purpose of this article is to analyze execution errors in the construction of a residential building in reinforced concrete structures with precast concrete slabs and masonry fencing, due to the incompatibility between projects. For the development of the research, monitoring of the construction phases was developed through regular technical visits and photographic recording. Being a small work, the projects were carried out independently and without prior study of the construction stages, which favored the non-conformities in this case study. Execution errors were found to be caused by incompatibility between architectural, structural, 
electrical and hydraulic projects that had a negative impact during the execution of the project. In this way, the importance of compatibility between projects is emphasized, regardless of the size of the construction.

Keywords: Compatibility. Construction. Incompatibility. Project.

\section{Introdução}

Com a modernidade da engenharia e tecnologia, os projetos que não possuem interdependência são desenvolvidos simultaneamente, para tanto, é necessária uma equipe de desenvolvimento, para que haja maior compatibilidade entre os projetos. Melhado (2013) denomina esse processo é por engenharia simultânea, onde esse processo de concepção busca valorizar a fase de elaboração e diminuir as incompatibilidades.

Apesar das afirmações positivas de Callegari (2007) referentes a utilizações de programas de unificação de projetos no processo de compatibilização, o uso de ferramentas que facilitam esse processo faz com que muitos profissionais passem despercebidos em relação as inconformidades dos diversos projetos de uma obra, não visualizando os detalhes e compatibilidade dos projetos como um todo, ocasionando inúmeros problemas construtivos, custos não previstos na planilha orçamentária e até mesmo atrasos no tempo final da obra.

Conforme Nunes et al. (2020) o País vive uma grande crise em todos âmbitos comerciais, sendo o momento atual da economia brasileira uma oportunidade propícia para transformações de postura deste setor, em particular na fase de transição entre projetos e construção. O presente trabalho abordou problemas construtivos gerados na execução devido à ausência de compatibilização de projetos, identificados na fase de execução de uma obra residencial, tendo como objetivo principal analisar erros de execução na construção em estrutura de concreto armado com laje pré-moldadas de concreto e vedação em alvenaria.

\section{Referencial Teórico}

A competitividade do mercado da construção civil é crescente, apresentando empreendimentos imobiliários cada vez mais complexos, necessitando de projetos primorosos que requerem um considerável esforço intelectual. Para tanto, Manenti (2018) recomenda uma articulação eficiente entre os processos da equipe multidisciplinar envolvida, remete às construtoras, empreendedores, projetistas e fornecedores da cadeia construtiva, uma mudança de postura tanto em relação aos processos de produção, como nos processos gerencias.

Nascimento (2014) afirma que os desenvolvimentos dos diversos projetos necessários para a construção de um edifício devem ser vistos como investimentos e não como custos extras, visto que é nesta fase onde se pode verificar dificuldades de execução, limitando causas indesejáveis posteriores, tais como, retrabalhos e atrasos.

Os benefícios da compatibilização de projetos são vistos tanto na execução da obra quanto na planilha financeira, Nascimento (2014) afirma que através da compatibilização é possível a redução dos custos, resolução de problemas, otimização das equipes de trabalho assim como a diminuição do consumo de insumos. Nobrega (2017) afirma e complementa dizendo que é na fase de projetos que as alterações devem ser feitas para evitar grandes custos.

$\mathrm{Na}$ concepção de um projeto os principais motivos que levam à incompatibilidade são a dificuldade de comunicação entre os profissionais envolvidos. Costa (2010) afirma que a interação entre todos os profissionais no processo de concepção do projeto é fundamental às informações e a sua apresentação. Na pesquisa feita por Nascimento (2014), os participantes entrevistados afirmaram como são comuns os problemas por ausência de compatibilização entre projetos, relatando a facilidade de se encontrar erros de execução, ressaltando as interferências entre projeto estrutural e instalações, assim como os projetos de arquitetura e drenagem. 
A definição de compatibilização de projetos não está especificada em uma metodologia única, porém alguns autores trazem definições. Melhado (2005) afirma que compatibilização são as necessidades de verificar as interferências para diminuir a problemática na execução e unir todos projetos em um único. Para Mikaldo (2006) a compatibilização é a interação entre diversos projetos, sendo a sobreposição de projetos 2D, integração de modelos 3D e outros métodos como FMEA (Análise do Modo e Efeito de Falha), podem ser utilizados com a finalidade de verificar incoerências entre projetos.

O meio de compatibilização atual é por intermédio metodologia Building Information Modeling (BIM), ela permite que os projetistas trabalhem de forma simultânea e interligada ao mesmo projeto. Crespo (2007) afirma que a utilização de ferramentas com a metodologia BIM possibilita orientar e gerenciar as informações da construção no ciclo completo do projeto.

\section{Metodologia}

Esta seção apresentará a metodologia utilizada para verificar, por meio de estudo de caso, o impacto da ausência de compatibilização na execução de uma obra residencial de alto padrão localizada no município de Porto Velho, capital do estado de Rondônia.

Nesta pesquisa foi estudada a execução de uma obra de edificação residencial por meio da coleta de dados para análises visuais e técnicas, seguido de anotações e registros fotográficos para descrever as incompatibilidades de projetos identificadas na fase de execução, a fim de propor melhorias para execução do projeto, tendo em vista tais incompatibilidades.

Para o desenvolvimento da pesquisa foi necessário primeiramente a seleção das obras em fase de execução, para realização de estudo de caso abrangendo as seguintes etapas; infraestrutura, superestrutura, vedação, instalações e complementares. Para ser realizado o levantamento fotográfico e documentação descritiva para análise da compatibilização, verificação e análise da incompatibilidade (interferências físicas), através de conflitos geométricos e de funcionalidade, acompanhamento no canteiro de obra, através de observações in loco e estudos de documentos com objetivo de verificar possíveis incompatibilidades entre os projetos de arquitetura e complementares que deram origem a modificações no projeto e/ou retrabalhos.

A obra estudada nesta pesquisa é referente a uma edificação residencial de concreto armado de um pavimento com área total construída de $234,39 \mathrm{~m}^{2}$. A coleta de dados foi feita presencialmente através de relatórios fotográficos e anotações técnicas no período de maio a agosto de 2019.

\section{Resultados}

Serão apresentados os resultados das incompatibilidades encontradas durante a execução da obra:

A primeira incompatibilidade é entre o projeto estrutural e instalações sanitárias. Antes da execução das instalações sanitárias houve a execução das vigas baldrames da residência, porém no projeto estrutural, na planta de formas e armações das vigas baldrames da edificação, não foram previstas as passagens dos tubos da rede sanitária. A execução foi realizada exatamente como solicitada no projeto estrutural, porém, posteriormente, para execução da passagem das tubulações sanitárias ocorreu a necessidade de perfurar algumas das vigas baldrames com o uso de martelete elétrico. O processo ocorreu em todos os banheiros da residência (Figura 1). 

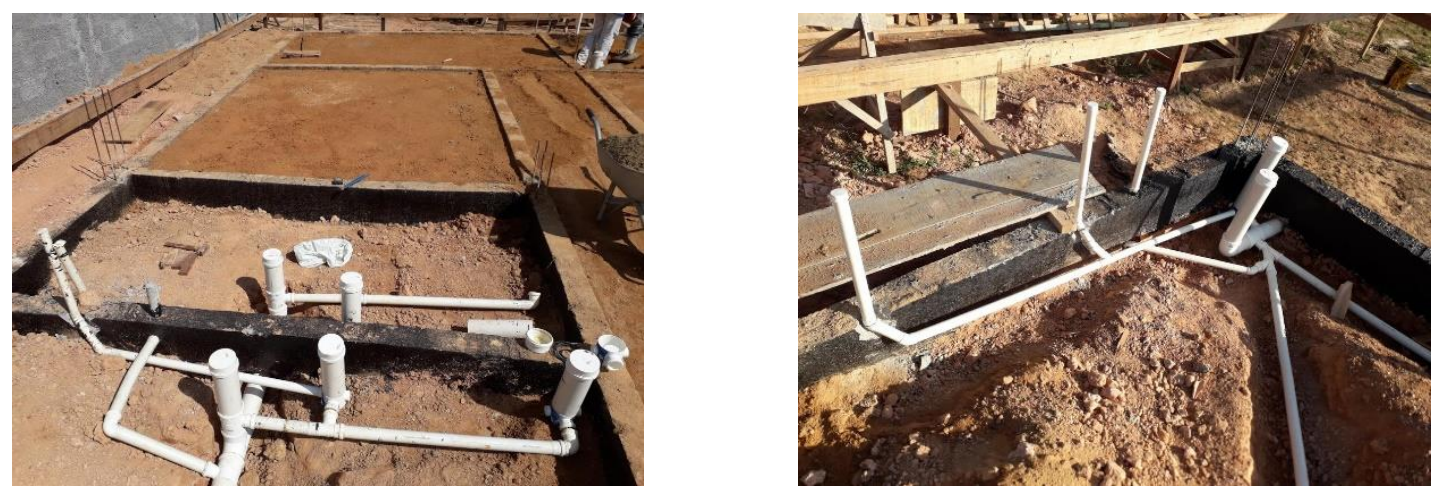

Figura 1 - Incompatibilidades entre projetos estrutural e sanitário.

O processo construtivo poderia ter ocorrido de maneira diferente caso, anteriormente, na etapa de criação do projeto houvesse uma compatibilização do projeto estrutural com o projeto de instalações sanitárias, pois fazendo a sobreposição entre o projeto estrutural e sanitário seria possível visualizar as interferências físicas entre as vigas baldrames com a rede de sanitária dos banheiros da edificação.

Apesar das precauções dos engenheiros na execução das vigas baldrames, foram inevitáveis os reajustes após a conclusão do processo executivo da infraestrutura da edificação, causando uma incompatibilidade entre o projeto estrutural e arquitetônico. Entre eles foi notado, conforme mostrado na Figura 2, que a parede interna da edificação não acompanhava corretamente o prolongamento da viga baldrame, devido a um recuo da parede no projeto de arquitetura. Neste caso foi necessária a execução de uma viga sobreposta a baldrame acima do nível do contra piso.

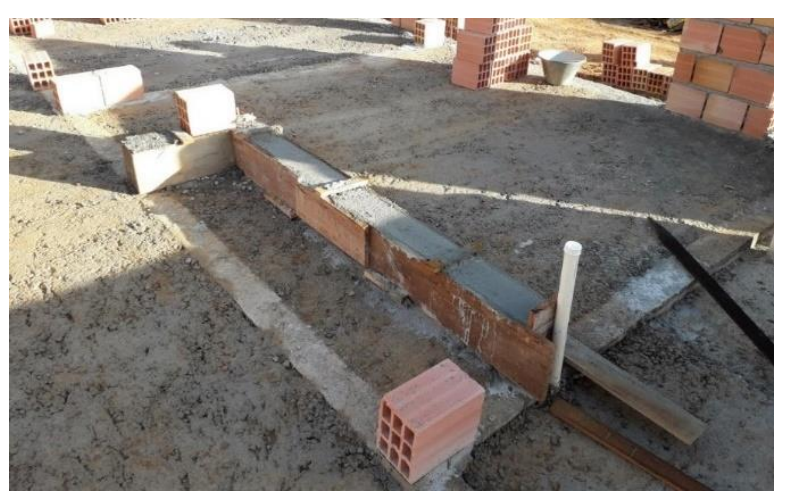

Figura 2 - Incompatibilidades entre projetos estrutural e arquitetônico.

Posterior à execução das vigas baldrames deu-se início à etapa de vedação da edificação e analisando as informações do projeto arquitetônico foi possível visualizar a divergência entre o projeto estrutural e projeto arquitetônico, onde por motivos técnicos executaram a viga baldrame não prevista em projeto sobreposta ao contra piso, caso o processo de análise de projeto houvesse ocorrido antes da execução, sendo realizado durante a criação dos projetos complementares seria provável a notoriedade da divergência entre projeto arquitetônico e estrutural.

Durante a visita técnica foi observado uma incompatibilidade que ocorreu entre o projeto estrutural e instalações elétricas. O mestre de obras executou a passagem dos eletrodutos através da armação do pilar para mitigar os efeitos da incompatibilidade (Figura 3). Neste caso, a posição do ponto de acionamento estava locada no projeto de instalações elétricas na mesma locação do pilar, sendo executado conforme indicado no projeto, porém, posteriormente, durante a visita técnica do engenheiro responsável pela execução da obra, o mesmo solicitou que o ponto fosse removido, pois a fixação do eletroduto dentro da armação do pilar diminuiria a sessão de concreto. 


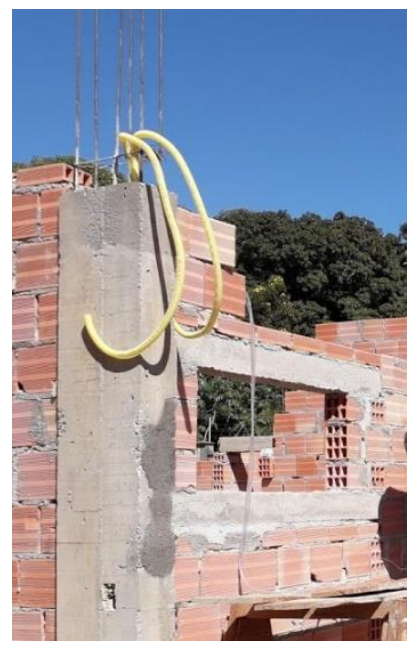

Figura 3 - Incompatibilidades entre projetos estrutural e elétrico (pilar).

Também foi verificada outra incompatibilidade entre os projetos estrutural e elétrico durante a execução das vigas baldrame do muro. Durante a passagem dos eletrodutos de alimentação elétrica da residência ocorreu a necessidade de passar condutos através de uma viga já concretada já concretado, porém, não havia os furos necessários para tal passagem. Desta maneira, foi necessária a demolição parcial do baldrame para executar a passagem dos condutos (Figura 4).

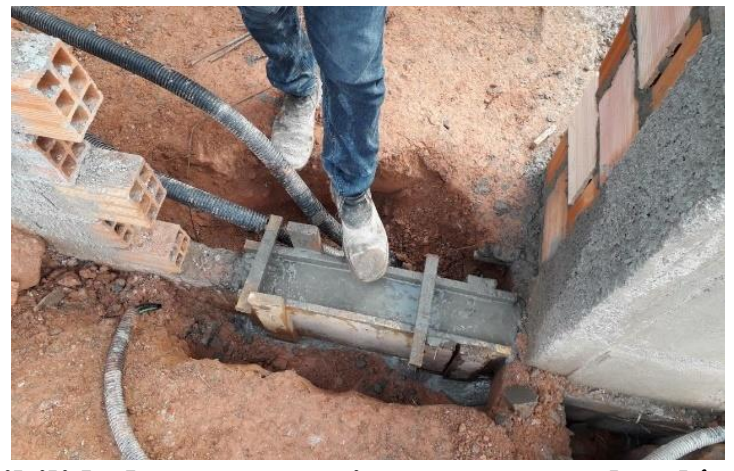

Figura 4 - Incompatibilidades entre projetos estrutural e elétrico (viga baldrame).

Caso houvesse uma compatibilização entre os dois projetos, durante o processo de execução não teria maiores problemas, pois seria possível executar as passagens necessárias na viga para os condutos de alimentação da rede elétrica da residência.

\section{Discussões}

Referente ao primeiro caso citado de incompatibilidade entre projeto estrutural e instalações sanitárias, o processo construtivo poderia ter ocorrido de maneira diferente caso, anteriormente, na etapa de criação de projeto houvesse uma compatibilização do projeto estrutural com o projeto de instalações sanitárias, pois fazendo a sobreposição entre o projeto estrutural e sanitário conforme Figuras 5 e Figura 6, é possível visualizar as interferências físicas entre as vigas baldrame com a rede de sanitária dos banheiros da edificação. 


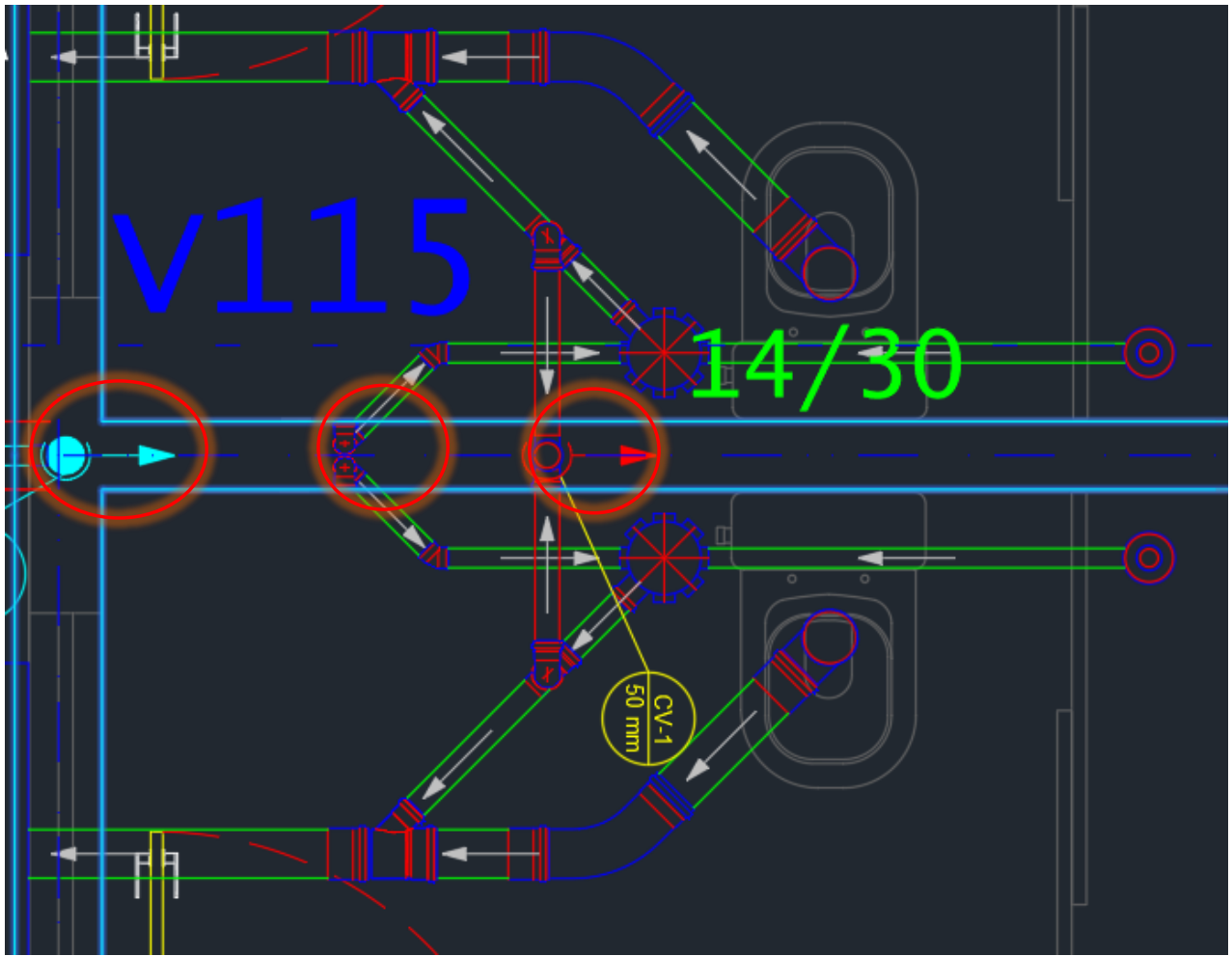

Figura 5 - Incompatibilidades estrutural e sanitário.

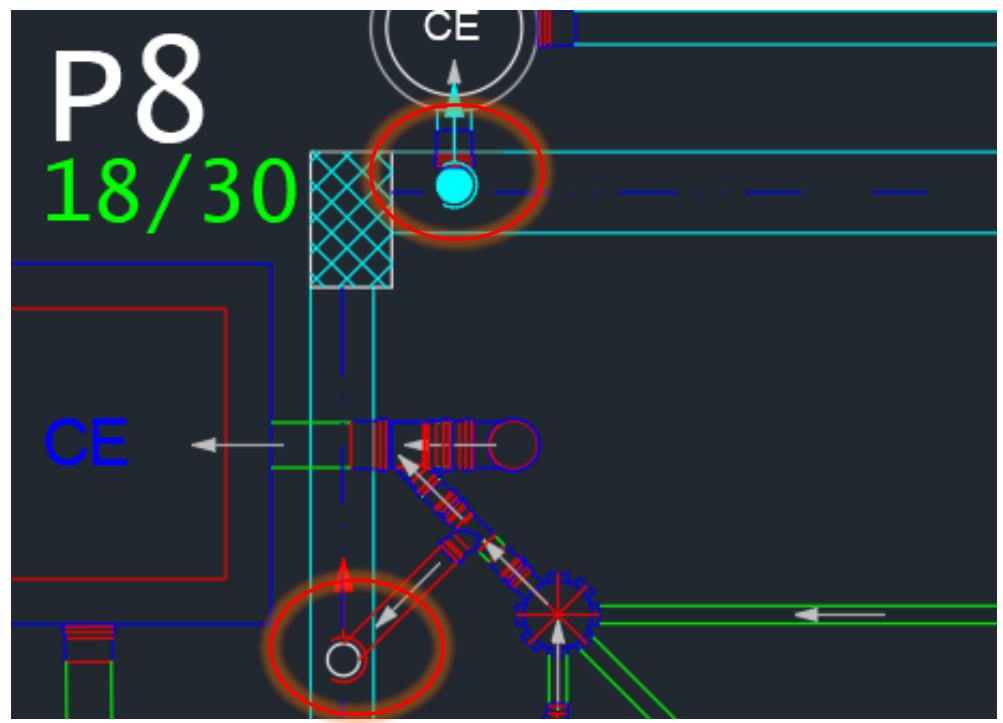

Figura 6 - Sobreposição entre projetos estrutural e hidráulico.

Conforme mostrado, com a compatibilização de projetos e possível visualizar as interferências executivas e físicas, mesmo na fase de projeto, utilizando a sobreposição em $2 \mathrm{D}$ conseguimos visualizar pelo menos cinco interferências das tubulações da rede sanitária com as vigas baldrames do projeto estrutural.

Analisando a incompatibilidade entre projetos estrutural e arquitetônico, juntamente com as informações dos projetos, é possível visualizar a divergência entre eles, onde, por motivos técnicos executaram a viga baldrame não prevista em projeto sobreposto ao contra piso. Se o processo de análise de projeto houvesse ocorrido antes da execução, sendo realizado durante a criação dos projetos complementares seria provável a notoriedade de incompatibilidade. Usando o método de sobreposição de projetos em 2D é possível visualizar, conforme a Figura 7 o desalinhamento da parede arquitetônica com a infraestrutura da edificação. 


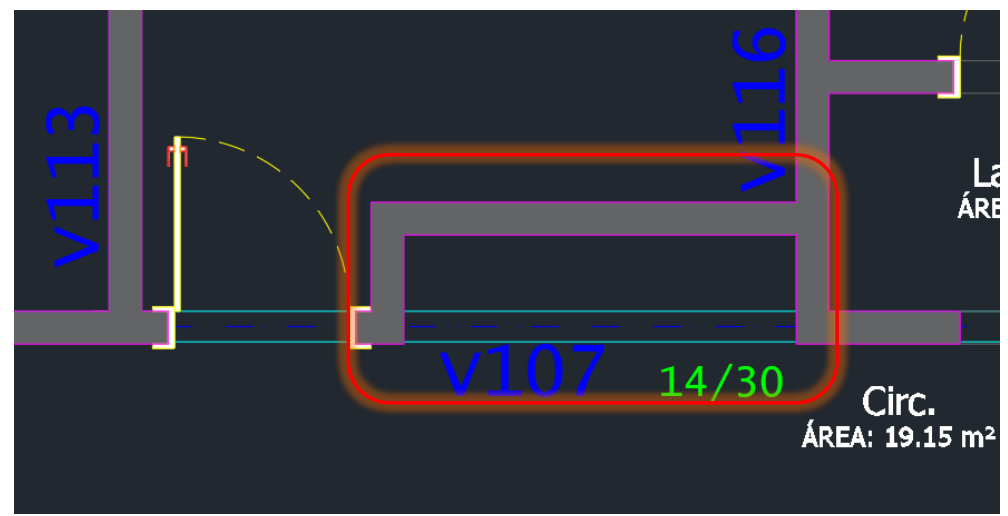

Figura 7 - Sobreposição entre projetos estrutural e arquitetônico.

O projeto estrutural do caso foi confeccionado individualmente por uma empresa de engenharia exclusiva da área de estruturas, o projeto em questão foi executado e entregue diretamente a empresa responsável pela execução sem passar pela etapa de compatibilização.

Conforme observado na Figura 8, a incompatibilidade entre projeto estrutural e instalações elétricas, foi um erro de execução, apesar do profissional responsável pelo acompanhamento da obra seguir rigorosamente o projeto. Todavia, o responsável pela execução do projeto de instalações elétricas houvesse feito a compatibilização do seu projeto com os demais, poderia ter visualizado a sobreposição entre o ponto de acionamento de iluminação com a posição de um pilar estrutural.

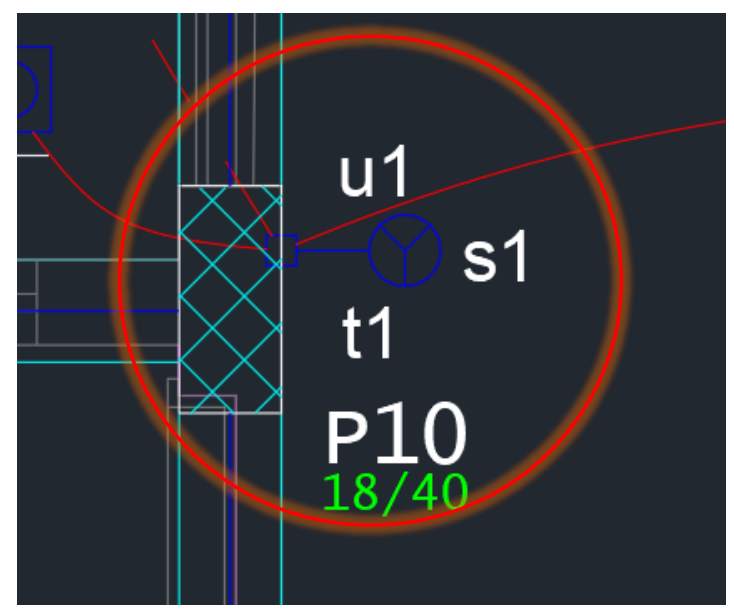

Figura 8 - Sobreposição entre projetos estrutural e elétrico.

Analisando os projetos estrutural e as instalações elétricas, é notória com a utilização da sobreposição de projetos, visualizar a passagem dos eletrodutos transversalmente a viga V121 (Figura 9), caso houvesse uma análise prévia dos projetos, no processo de compatibilização o processo construtivo poderia ter ocorrido de maneira mais coerente, evitando o retrabalho tornando a obra mais ordenada diminuindo os custos desnecessários. 


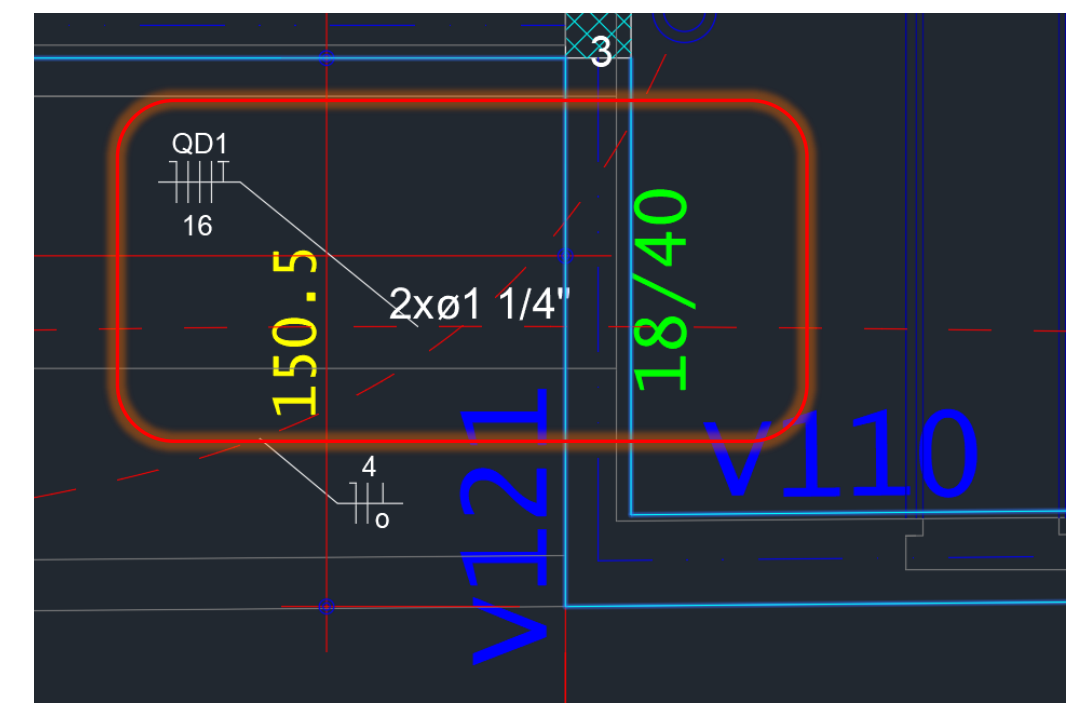

Figura 9 - Sobreposição entre projetos estrutural e elétrico.

Utilizando a sobreposição em 2D conseguimos fazer a compatibilização que possibilitou a visualização das interferências entre o projeto estrutural e o projeto de instalações elétricas. Desta maneira é presumível as incompatibilidades físicas entre o projeto estrutural e o projeto de instalações elétricas.

\section{Conclusões}

A partir da pesquisa desenvolvida conclui-se que alguns erros de execução causando retrabalhos e desperdícios estão relacionados com a ausência de compatibilização entre projetos e sendo uma obra de pequeno porte, os projetos foram realizados de maneira independente e sem o estudo prévio das etapas da construção o que favoreceu as inconformidades neste estudo de caso. Mesmo que ocorra a análise de projetos durante a execução é quase inevitável o retrabalho ou a execução de serviços não previstos anteriormente.

Contudo, fica evidenciada a importância da compatibilização de projetos, mesmo em obra de pequeno porte, sendo notória que a compatibilização de projetos é uma etapa essencial da fase de planejamento e execução dos projetos, não sendo totalmente eficaz durante a fase de execução.

\section{Agradecimentos}

Os autores agradecem à Universidade Federal de Rondônia (UNIR) e à sua Pró-Reitoria de Pós-Graduação e Pesquisa (PROPESQ).

\section{Referências}

Callegari, S. (2007). Análise da compatibilização de projetos em três edifícios residenciais multifamiliares. Trabalho de Conclusão de Curso, Universidade Federal de Santa Catarina, Florianópolis, SC, Brasil.

Costa, L. D. M. (2010). Compatibilização de projetos e gerenciamento de resíduos como condições primordiais para a sustentabilidade das construções. Trabalho de Conclusão de Curso, Universidade Federal de Santa Catarina, Florianópolis, SC, Brasil.

Crespo, C. C. (2007). Ferramentas BIM: um desafio para a melhoria no ciclo de vida do projeto. Trabalho de Conclusão de Curso, Universidade de Campinas, Campinas, SP, Brasil.

Manenti, E. M. (2018). Diretrizes para elaboração do plano de execução bim para contratos de projetos de edificações. Dissertação de Mestrado, Universidade Federal de Santa Catarina, Florianópolis, SC, Brasil. 
Melhado, S. M. (2005). Qualidade de projeto na construção de edifícios: aplicação ao caso das empresas de incorporação e construção. Tese de Doutorado, Universidade de São Paulo, São Paulo, SP, Brasil.

Mikaldo, J. R. J. (2006). Estudo comparativo do processo de compatibilização de projetos em $2 D e$ $3 D$ com uso de TI. Dissertação de Mestrado, Universidade Federal do Paraná, Curitiba, PR, Brasil.

Nascimento, J. M. A. (2014). A importância da compatibilização de projetos como fator de redução de custos na construção civil. Revista Especialize IPOG, 1(7), 1-11.

Nobrega, U. R. G. (2017). A importância da compatibilização de projetos das edificações para minimizar as falhas na execução, reduzir custos e garantir um maior controle de qualidade. Trabalho de Conclusão de Curso, Universidade Federal da Paraíba, João Pessoa, PB, Brasil.

Nunes, J. M., Longo, O. C., Alcoforado, L. F., \& Pinto, G. O. (2020). The civil construction sector in Brazil and the current economic crisis. Research, Society and Development, 9(9), e393997274. https://doi.org/10.33448/rsd-v9i9.7274 